\title{
Collodion Baby with TGMI gene mutation
}

This article was published in the following Dove Press journal:

International Medical Case Reports Journal

22 September 2015

Number of times this article has been viewed

\section{Deepak Sharma' \\ Basudev Gupta ${ }^{2}$ \\ Sweta Shastri ${ }^{3}$ \\ Aakash Pandita' \\ Smita Pawar ${ }^{4}$ \\ 'Department of Neonatology, Fernandez Hospital, Hyderguda, Hyderabad, Andhra Pradesh, \\ ${ }^{2}$ Department of Pediatrics, \\ Civil Hospital, Palwal, Haryana, \\ ${ }^{3}$ Department of Pathology, NKP Salve \\ Medical College, Nagpur, Maharashtra, \\ ${ }^{4}$ Department of Obstetrics and \\ Gynaecology, Fernandez Hospital, \\ Hyderguda, Hyderabad, Andhra \\ Pradesh, India}

Correspondence: Basudev Gupta

Department of Pediatrics, Civil Hospital,

Palwal, Haryana, India

Tel +9l 9416738222

Email drbdgupta78@gmail.com
Abstract: Collodion baby (CB) is normally diagnosed at the time of birth and refers to a newborn infant that is delivered with a lambskin-like membrane encompassing the total body surface. $\mathrm{CB}$ is not a specific disease entity, but is a common phenotype in conditions like harlequin ichthyosis, lamellar ichthyosis, nonbullous congenital ichthyosiform erythroderma, and trichothiodystrophy. We report a CB that was brought to our department and later diagnosed to have TGM1 gene c.984+1G >A mutation. However, it could not be ascertained whether the infant had lamellar ichthyosis or congenital ichthyosiform erythroderma (both having the same mutation). The infant was lost to follow-up.

Keywords: cellophane membrane, c.984+1G >A mutation, lamellar ichthyosis, nonbullous congenital ichthyosiform erythroderma, parchment membrane, TGM1 gene

\section{Introduction}

Collodion baby (CB) is an uncommon dermatological condition, and is a common manifestation of various hereditary disorders including harlequin ichthyosis, lamellar ichthyosis (LI), nonbullous congenital ichthyosiform erythroderma (NBCIE), and trichothiodystrophy metabolic diseases and endocrine diseases. $\mathrm{CB}$ is an extremely rare condition with an estimated incidence of one in 50,000 to 100,000 deliveries. ${ }^{1,2} \mathrm{We}$ report a $\mathrm{CB}$ that was brought to our department and was diagnosed to have TGM1 gene c.984+1G $>$ A mutation. However, we could not confirm whether the patient had LI or NBCIE as both these conditions share the same mutation and the infant was later lost to follow-up. Although a few cases with this mutation have been reported from around the world, ${ }^{3,4}$ this was the first case of its type to be reported from our country.

\section{Case presentation}

A term, male neonate was delivered by spontaneous vaginal delivery to a primigravida mother with the parents being non-consanguineous. The infant had a birth weight of $3.5 \mathrm{~kg}$ (appropriate for gestational age), with length and head circumference being 52 and $34 \mathrm{~cm}$, respectively. The Apgar score was normal: 8/9/9 at 1, 5, and 10 minutes, respectively. Within hours of birth, the newborn was referred to our emergency department in view of a parchment-like membrane covering the whole body. A detailed physical examination showed broad, thick scales all over the body with predominance over the chest, abdomen, and upper arms. There was associated ectropion and eclabion too. An excess of scales around the mouth gave the typical fish-like mouth appearance. The infant also had generalized erythema and edema over the body (Figure 1). There were no other associated obvious anomalies. Detailed 


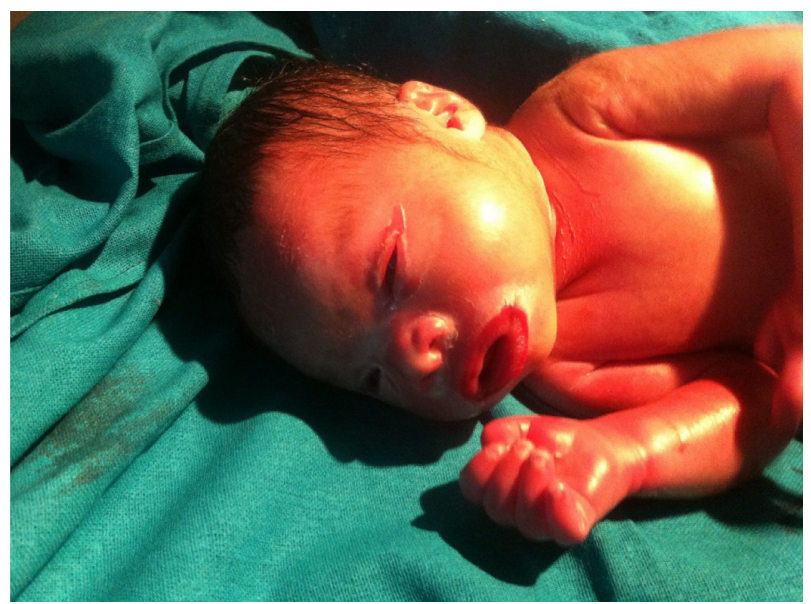

Figure I Parchment-like membrane covering the whole body with predominance over the chest, abdomen, and upper limbs.

Note: There is excess of scales around the mouth giving appearance of fish-like mouth.

systemic examination was carried out which revealed no abnormalities. Family history was taken and a pedigree analysis was done. There was no history of consanguinity or dermatological-related disorders in the family. There was no history of maternal complications during pregnancy or maternal drug exposure. Dermatological opinion was sought and the infant was managed with adequate humidification in a high-humidity incubator (relative humidity $80 \%$ ). Close monitoring of body temperature and adequate fluid and electrolyte replacement was done. Oral feeds by tube were started at age of 1 day and gradually increased to full feeds by age of 2 days. Tube feeds were continued for 1 week followed by expressed breast milk by spoon. Nutritionist opinion was also taken for adequate calorie supplementation and fortification of the expressed breast milk was done accordingly. Breastfeeding was tried on several occasions but was difficult due to the collodion membrane around the mouth. Thus, spoonfeeding was continued until the age of 3 weeks, and once the membranes resolved the infant was put on breast-feeding. Topical emollients were regularly applied as advised by the dermatologist. Ophthalmological opinion was taken for eye care and artificial tears were applied to prevent drying of eyes. The infant was closely monitored for signs and symptoms of sepsis in the form of off color, shock, and delayed perfusion. The scales resolved over the next 3 weeks and the infant was discharged in well condition at the age of 4 weeks. A skin-punch biopsy was done at the age of 3 weeks from the abdominal skin. The hematoxylin-eosin stain revealed markedly thickened stratum corneum with cholesterol clefts and a normal granular layer. Geneticist consultation was taken and, in view of financial constraints, gene analysis for only $T G M 1$ gene mutation was sent, it being the commonest cause of $\mathrm{CB}$. Targeted gene sequencing was performed on the infant's blood and the DNA isolated was used to perform exome capture using custom capture kit. The genetic analysis revealed a homozygous mutation in transglutaminase-1 (TGM1 gene p.Val330MetfsX12 [c.984+1G >A]) located on chromosome 14q12. Genetic analysis of the parents was not performed, again due to financial constraints. Consent was obtained from the parents for case reporting and image publication. However, the infant was later lost to follow-up and, thus, we were unable to do long-term follow-up and it could not be ascertained whether the infant had LI or NBCIE. Both these conditions have the same mutation and only long-term skin manifestations can help to differentiation between the two. Written informed consent was obtained from parents for the publication of images and the case report. All principles outlined in the Declaration of Helsinki were followed.

\section{Discussion}

The term "collodion baby", also known sometimes as "collodion foetus", was first used in 1884 to describe the physical appearance of a newborn with a parchment-like membrane covering the whole body. ${ }^{3}$ Collodion membrane is described in literature as a congenital, transient keratinized membrane that is usually secondary to a disorder of cornification. ${ }^{4}$ It is usually parchment- or cellophane-like, shiny and taut, and wraps over whole of the neonate's body. These babies are usually born prematurely and are often diagnosed at the time of birth. ${ }^{5}$

The tight collodion membrane around the whole body causes many complications like ectropion; ${ }^{6}$ eclabium; pseudo-contractures of limbs leading to restricted extension of the digits and extremities; absence of eyebrows; and hypoplasia of nasal and auricular cartilage with flattening of the ears and nostrils. ${ }^{7}$ This membrane also often leads to poor sucking; restricted pulmonary function; digital vascular constriction; ${ }^{8}$ distal limb ischemia; hypohydrosis; and edema of the extremities. ${ }^{9}$ Infants usually have hypothermia or hyperthermia; hypohydrosis; excessive insensible water loss leading to hypernatremic dehydration; failure to thrive secondary to poor feeding; and infections, both cutaneous and systemic like candida and bacterial. After birth, the membrane starts drying and breaks down completely as a result of chest expansion. Tests for confirmation of diagnosis can only be performed after the membrane has completely shed off. ${ }^{10} \mathrm{CB}$ is the phenotype of many conditions and can be read from many excellent reviews. ${ }^{6,11-13}$

A disease severity score has been developed for newborns with collodion membrane based on the various physical 
parameters to forecast the outcome and ramifications in these infants. ${ }^{13}$ There has been a case report of CB secondary to ingestion of drugs like infliximab. ${ }^{14}$ The biopsy of the skin is nonspecific and shows compact and thickened stratum corneum. ${ }^{15}$

LI (MIM \#242300) is a member of the autosomal recessive congenital ichthyosis family that is characterized by disorders of keratinization and is distinguished as a non-syndromic form of ichthyosis. The incidence of occurrence of LI has been estimated at one case per $138,000-300,000$ population. In the recent past, few other syndromes were also included in the autosomal recessive congenital ichthyosis family, like bathing suit ichthyosis, harlequin ichthyosis, astral self-healing, and self-healing CB. ${ }^{16}$ The bulk of LI is caused by mutations in one of various genes, including TGM1, NIPAL4, ALOX12B, ALOXE3, ABCA12, CYP4F22, PNPLA1 (OMIM 615024), ${ }^{17}$ and CERS3 (OMIM 615023). TGM1 is located on chromosome 14q11.2 and has 15 exons (GenBank NM-000359.2). ${ }^{18}$

In LI, the collodion membrane is normally replaced by thick hyperkeratotic membrane with dark, shell-like adherent scales. The histopathological examination of skin shows cholesterol clefts in the stratum corneum, and absence or thinning of cornified envelope with small keratohyalin granules and lipid droplets. At birth, electron microscopy can be used to differentiate a severe CB affected by LI from a baby affected by harlequin ichthyosis by demonstrating the absence of the marginal band. ${ }^{15}$ There has been a case report of LI with rickets, carcinoma of the hypopharynx, and celiac disease, ${ }^{19}$ thus demonstrating the importance of regular follow-up. ${ }^{20}$

NBCIE is an autosomal recessive disorder that usually has mild clinical features. It has recently been shown that NCIE results because of mutations in $A L O X 12 B$ and TGM1 genes resulting in production of abnormal TGM1. The clinical features include congenital erythroderma and scaling. The other parts involved include face, flexural sites, palms, and soles, but ectropion is usually not seen. Hair is usually sparse and nails dystrophic. Pediatric patients with NCIE disorder have erythema with finer, white scales. These patients also have cutaneous infections with bacteria, yeasts, and dermatophytes. The skin biopsy is nonspecific with a normal granular layer. Prenatal diagnosis is possible if there is history of NCIE in previous pregnancy. ${ }^{21}$

The prognosis of CB depends on the associated disorders and complications in the neonatal period and the genetic mutation responsible for $\mathrm{CB}$. These patients need regular follow-up and the parents should be counseled for the expected complications. ${ }^{8}$
Management of neonates with collodion usually requires a multidisciplinary approach that includes the parents, supporting staff, neonatologists, dermatologists, and, in some cases, ophthalmologist and otorhinolaryngologist. The primary aim of treatment in CB is to eliminate scaling and to reduce xerosis without causing excessive irritation. Prior to deciding on treatment, various factors should be considered such as age and sex of the patient, type and severity of the disease, and the extent and site of the lesions. ${ }^{4}$

There is a high risk of dehydration and electrolyte imbalances, because of high insensible water loss from the membrane. This requires placement in a high-humidity incubator and close monitoring of body temperature as well as also good nutrition support, both in enteral and parenteral forms. ${ }^{22,23}$ Moisturizers and topical keratolytic agents are usually the first-line therapeutic options in management. They improve skin barrier function and facilitate desquamation. The various options available as moisturizers and lubricants include sodium chloride, urea, vitamin E acetate, glycerol, and petroleum jelly. In infants and children with thick scaling and marked hyperkeratosis, one or more keratolytic agents, such as lactic acid, glycolic acid, salicylic acid, $\mathrm{N}$-acetyl-cysteine, urea $(>5 \%)$, or propylene glycol, can be added. Ectropion can be managed with the application of artificial tears and eye lubricants. Surgical correction is reserved for severe cases. ${ }^{24}$ Oral retinoids have keratolytic effects; they help in elimination of scales and prevent excessive hyperkeratosis. Both isotretinoin and aromatic retinoids (acitretin and etretinate) have been proved to be effective in treatment. Regular cleansing of the external auditory canal by an ear-throat-nose specialist can prevent scales from accumulating and so prevent hearing loss. ${ }^{25}$

\section{Conclusion}

$\mathrm{CB}$ is a newborn with a parchment- or cellophane-like membrane covering the whole body surface. The diagnosis of $\mathrm{CB}$ requires a detailed family history, skin biopsy, and genetic analysis for confirmation. Skin biopsy forms an important tool for diagnosis of the cause of $\mathrm{CB}$ and specific biopsy features help in reaching the correct diagnosis. It should be done before genetic analysis but after the membrane is shed off. Prenatal diagnosis should be offered to parents who have had previous affected children so that antenatal counseling can be done and an option for termination given. The prognosis depends on the associated genetic mutation leading to $\mathrm{CB}$. These infants need regular follow-up, as they are prone for long-term complications. 


\section{Acknowledgment}

There was no honorarium, grant, or other form of payment given to anyone to produce the paper.

\section{Disclosure}

The authors report no conflicts of interest in this work.

\section{References}

1. Dyer JA, Spraker M, Williams M. Care of the newborn with ichthyosis. Dermatol Ther. 2013;26(1):1-15.

2. Dreyfus I, Chouquet C, Ezzedine K, et al. Prevalence of inherited ichthyosis in France: a study using capture-recapture method. Orphanet $J$ Rare Dis. 2014;9:1.

3. Herman ML, Farasat S, Steinbach PJ, Wei MH, Toure O, Fleckman P, Blake P, Bale SJ, Toro JR. Transglutaminase-1 gene mutations in autosomal recessive congenital ichthyosis: summary of mutations (including 23 novel) and modeling of TGase-1. Hum Mutat. 2009;30(4):537-47.

4. Fachal L, Rodríguez-Pazos L, Ginarte M, Beiras A, Suárez-Peñaranda JM, Toribio J, Carracedo Á, Vega A. Characterization of TGM1 c. $984+1 \mathrm{G}>$ A mutation identified in a homozygous carrier of lamellar ichthyosis. Int J Dermatol. 2012;51(4):427-30.

5. Ivich JM. Ichthyosis in the Neonatal Setting. Adv Neonatal Care. 2015; 15(4):253-260.

6. Panse N, Sahasrabudhe P. Ectropion in a case of collodion baby. World J Plast Surg. 2014;3(2):146-147.

7. Akcakus M, Gunes T, Kurtoglu S, Ozturk A. Collodion baby associated with asymmetric crying facies: a case report. Pediatr Dermatol. 2003;20(2):134-136.

8. Taïeb A, Labrèze C. Collodion baby: what's new. J Eur Acad Dermatol Venereol. 2002;16(5):436-437.

9. Mazereeuw-Hautier J, Aufenvenne K, Deraison C, et al. Acral selfhealing collodion baby: report of a new clinical phenotype caused by a novel TGM1 mutation. Br J Dermatol. 2009;161(2):456-463.

10. Prado R, Ellis LZ, Gamble R, Funk T, Arbuckle HA, Bruckner AL. Collodion baby: an update with a focus on practical management. J Am Acad Dermatol. 2012;67(6):1362-1374.

11. DiGiovanna JJ, Robinson-Bostom L. Ichthyosis: etiology, diagnosis, and management. Am J Clin Dermatol. 2003;4(2):81-95.

12. Schmuth M, Martinz V, Janecke AR, et al. Inherited ichthyoses/ generalized Mendelian disorders of cornification. Eur J Hum Genet. 2013;21(2):123-133.
13. Rubio-Gomez GA, Weinstein M, Pope E. Development of a disease severity score for newborns with collodion membrane. J Am Acad Dermatol. 2014; 70(3):506-511.

14. Offiah M, Brodell RT, Campbell LR, Wyatt JP. Collodion-like membrane in a newborn exposed to infliximab. J Am Acad Dermatol. 2014; 71(1):e22-e23.

15. Vahlquist A, Bygum A, Gånemo A, et al. Genotypic and clinical spectrum of self-improving collodion ichthyosis: ALOX12B, ALOXE3, and TGM1 mutations in Scandinavian patients. J Invest Dermatol. 2010; 130(2):438-443.

16. Rodríguez-Pazos L, Ginarte M, Vega A, Toribio J. Autosomal recessive congenital ichthyosis. Actas Dermosifiliogr. 2013;104(4):270-284. English, Spanish.

17. Fachal L, Rodríguez-Pazos L, Ginarte M, Carracedo A, Toribio J, Vega A. Identification of a novel PNPLA1 mutation in a Spanish family with autosomal recessive congenital ichthyosis. Br J Dermatol. 2014; 170(4):980-982.

18. Fachal L, Rodríguez-Pazos L, Ginarte M, et al. Characterization of TGM1 c. $984+1 \mathrm{G}>$ A mutation identified in a homozygous carrier of lamellar ichthyosis. Int J Dermatol. 2012;51(4):427-430.

19. Bubna AK, Veeraraghavan M, Anandan S, Rangarajan S. A case of lamellar ichthyosis with rickets and carcinoma of the hypopharynx. Indian J Dermatol. 2014;59(6):634.

20. Nenna R, D'Eufemia P, Celli M, et al. Celiac disease and lamellar ichthyosis. Case study analysis and review of the literature. Acta Dermatovenerol Croat. 2011;19(4):268-270.

21. Akiyama M, Sawamura D, Shimizu H. The clinical spectrum of nonbullous congenital ichthyosiform erythroderma and lamellar ichthyosis. Clin Exp Dermatol. 2003;28(3):235-240.

22. Buyse L, Graves C, Marks R, Wijeyesekera K, Alfaham M, Finlay AY. Collodion baby dehydration: the danger of high transepidermal water loss. Br J Dermatol. 1993;129(1):86-88.

23. Moskowitz DG, Fowler AJ, Heyman MB, et al. Pathophysiologic basis for growth failure in children with ichthyosis: an evaluation of cutaneous ultrastructure, epidermal permeability barrier function, and energy expenditure. J Pediatr. 2004;145(1):82-92.

24. Sigurdsson H, Baldursson BT. Inverting Sutures With Systemic Retinoids and Lubrication Can Correct Ectropion in Ichthyosis. Ophthal Plast Reconstr Surg. Epub 2014 Sep 11.

25. Harvey HB, Shaw MG, Morrell DS. Perinatal management of harlequin ichthyosis: a case report and literature review. J Perinatol. 2010; 30(1):66-72.
International Medical Case Reports Journal

\section{Publish your work in this journal}

The International Medical Case Reports Journal is an international, peer-reviewed open-access journal publishing original case reports from all medical specialties. Previously unpublished medical posters are also accepted relating to any area of clinical or preclinical science. Submissions should not normally exceed 2,000 words or

\section{Dovepress}

4 published pages including figures, diagrams and references. The manuscript management system is completely online and includes a very quick and fair peer-review system, which is all easy to use. Visit http://www.dovepress.com/testimonials.php to read real quotes from published authors. 